\title{
ALE-MHD TECHNIQUE FOR MODELING THREE-DIMENSIONAL MAGNETIC IMPLOSION OF A LINER
}

\author{
O. G. OLKHOVSKAYA, A. YU. KRUKOVSKY, YU. A. POVESCHENKO, \\ YU. S. SHAROVA*, AND V. A. GASILOV
}

Keldysh Institute of Applied Mathematics. Moscow, Russia.

*Corresponding author. E-mail: yulia-shar@mail.ru

\section{DOI: 10.20948/mathmontis-2021-50-11}

Summary. The article is devoted to the methodology for modeling current-carrying plasma in a Z-pinch studied in pulsed-power experiments. We discuss simulation performed via moving Lagrangian-Euler difference grid. The difference scheme approximating the hydrodynamic equations of a high-temperature medium possesses a "complete conservation" property and includes energy balances between the plasma components taking into account electromagnetic field - matter interaction and conductive (electronic, ionic) as well as radiative heat transfer. Numerical experiments provide quantitative estimates of physical effects which lead to essential distortions of a plasma shell during its magnetically-driven implosion. Performed simulations show the effect of instabilities on the final pinch structure, mainly, the hydrodynamic Rayleigh-Taylor instability and instability of a temperature-inhomogeneous plasma.

\section{INTRODUCTION}

Difference schemes of summarised approximation [1], schemes of physical splitting [2] and their modifications are widely used since they allow reproducing accurately specific feature of studied physical processes and perform calculations economically. This is why such schemes are widely used in application codes designed for multiphysics simulations (see e.g., $[3,4]$ ). In the monograph [5], algorithms for successive accounting physical processes are constructed for Lagrangian difference schemes of gasdynamics (GD) and magnetic gasdynamics (MHD). The advantage of the completely conservative difference schemes presented in [5] is the numerical approximation of the basic conservation laws (mass, momentum and energy) as well as additional balance equations, important for simulation of fast plasma-dynamic processes. These include the intensive energy transfer from external sources, the significant role of heat transfer by radiation, electron-ion energy relaxation, etc.

In this paper, we present a numerical technique based on the summarised approximation scheme developed for simulation of magnetoaccelerated plasma, primarily for various types of Z-pinches [6, 7].

Electrodynamic compression caused by the action of a current pulse on a plasma body in the form of a liner or a shell was first proposed for achieving thermonuclear fusion conditions [8]. Currently, such experimental schemes are mainly studied in order to generate soft x-ray radiation pulses via kinetic-to-thermal energy conversion at the stage of an accelerated shell collapse $[6,9,10]$. The development of high-current generators with the pulse duration about 100 nanoseconds and the peak current up to several MA, used in modern Z-pinch experiments, allows new prospects in this research area. High power soft x-ray sources up to several TW can be used not only in basic research, but also in industrial applications:

2010 Mathematics Subject Classification: 00A72, 65M06, 85D10.

Key words and Phrases: Magnetic Gas Dynamics, Plasma Dynamics, Completely Conservative Difference Scheme, Method of Combined Iterations, Gas-Puff Z-pinch. 
materials with gradient properties, x-ray photolithography, short-wave lasers etc. The problem of current-carrying plasma stabilization is actively studied. In the ongoing research, much attention is paid to the design (material and geometry) of imploding liners. Fitting of the liner parameters with the electrical parameters of the generator is important to ensure high efficiency transformation of the electricity into the kinetic energy of the liner [10-12].

The article consists of two parts.

The first part is devoted to the construction of Lagrangian-Eulerian numerical method and algorithms for solving the MHD in the form of conservation laws.

Implicit completely conservative difference scheme (CCDS) [1, 2] in Lagrangian variables is considered as a base. The Lagrangian-Eulerian approach provides opportunities for combining explicit and implicit approximations of convective flows.

The algorithm developed in this paper employs local splitting of physical processes. For two-dimensional MHD problems, similar algorithms were considered in [14]. The system of difference equations is divided into groups, and each group is solved by its own iterative process. Local iterative processes are assembled into an overall cycle, with the convergence controlled by the overall energy balance.

It is possible to remap computed Lagrangian values to a modified grid with mass, momentum, and total energy conservation. We also check the balances of internal and magnetic energy.

We use two-level temporal approximations of convective terms to ensure the CCDS for the numerical MHD system. Homogeneity of calculations for flows with strong discontinuities is achieved by introducing artificial viscosity [5], taking into account recommendations [15] for its adaptation to flow properties.

The second part of the paper gives an example of modeling the dynamics of a Z-pinch compression. The liner is formed during ablation and subsequent implosion of a wire array under the impact of a powerful current pulse. In computational experiments, we study typical instabilities that have the most significant effect on the magnetic implosion. Namely, we observe the hydromagnetic Rayleigh - Taylor instability along with instabilities of temperature-inhomogeneous plasma. The last may lead to matter overheating or radiative collapse depending on disbalance between Joule heating and radiation losses. The Conclusion summarizes the main results of the numerical experiments.

\section{GOVERNING MHD SYSTEM}

We use the common notation for the MHD equations in Cartesian coordinates: $t$ is the time, $\mathbf{U}=(u, w, v)$ is the substance velocity, $\rho$ is the density, $P$ is the gas pressure, $T$ is the temperature, $\varepsilon$ is the specific internal energy, $\mathbf{W}=\left(W_{x}, W_{y}, W_{z}\right)$ is the heat flux, $\mathbf{B}=\left(B_{x}, B_{y}, B_{z}\right)$ is the magnetic induction, $\mathbf{E}=\left(E_{x}, E_{y}, E_{z}\right)$ is the electric field strength, $\mathbf{j}=\left(j_{x}, j_{y}, j_{z}\right)$ is the electric current density, $c$ is speed of light in vacuum, $\sigma$ and $\kappa$ are the coefficients of electrical and thermal conductivity.

The kinematic equations for fluid particle positions:

$$
\frac{d x}{d t}=u ; \frac{d y}{d t}=w ; \frac{d z}{d t}=v ;
$$

The continuity equation: 


$$
\frac{d \rho}{d t}+\rho \frac{\partial u}{\partial x}+\rho \frac{\partial w}{\partial y}+\rho \frac{\partial v}{\partial z}=0
$$

The momentum equation projections on the coordinate directions:

$$
\begin{gathered}
\rho \frac{d u}{d t}=-\frac{\partial}{\partial x}\left(P+\frac{B^{2}}{8 \pi}\right)+\frac{1}{4 \pi}\left(\frac{\partial\left(B_{x}^{2}\right)}{\partial x}+\frac{\partial\left(B_{x} B_{y}\right)}{\partial y}+\frac{\partial\left(B_{x} B_{z}\right)}{\partial z}\right), \\
\rho \frac{d w}{d t}=-\frac{\partial}{\partial y}\left(P+\frac{B^{2}}{8 \pi}\right)+\frac{1}{4 \pi}\left(\frac{\partial\left(B_{x} B_{y}\right)}{\partial x}+\frac{\partial\left(B_{y}^{2}\right)}{\partial y}+\frac{\partial\left(B_{y} B_{z}\right)}{\partial z}\right), \\
\rho \frac{d v}{d t}=-\frac{\partial}{\partial z}\left(P+\frac{B^{2}}{8 \pi}\right)+\frac{1}{4 \pi}\left(\frac{\partial\left(B_{x} B_{z}\right)}{\partial x}+\frac{\partial\left(B_{y} B_{z}\right)}{\partial y}+\frac{\partial\left(B_{z}^{2}\right)}{\partial z}\right)
\end{gathered}
$$

The hydrodynamic pressure in (3) is equal to the sum of the partial pressures $P=P_{e}+P_{i}$.

The equations for the internal energies of the components:

$$
\begin{aligned}
& p \frac{d \varepsilon_{e}}{d t}=-\rho P_{e} \frac{\partial(1 / \rho)}{\partial t}-\frac{\partial\left(W_{e}\right)_{x}}{\partial x}-\frac{\partial\left(W_{e}\right)_{y}}{\partial y}-\frac{\partial\left(W_{e}\right)_{z}}{\partial z}+Q_{e i}+G_{j}+G_{e} \\
& p \frac{d \varepsilon_{i}}{d t}=-\rho P_{i} \frac{\partial(1 / \rho)}{\partial t}-\frac{\partial\left(W_{i}\right)_{x}}{\partial x}-\frac{\partial\left(W_{i}\right)_{y}}{\partial y}-\frac{\partial\left(W_{i}\right)_{z}}{\partial z}-Q_{e i}+G_{i}
\end{aligned}
$$

Here $G_{e, i}$ are sources (sinks) of electronic and ionic energy, $G_{j}$ is the mass energy density of the Joule heating:

$$
\begin{gathered}
G_{j}=\frac{1}{4 \pi}\left(E_{x} j_{x}+E_{y} j_{y}+E_{z} j_{z}\right) \\
j_{x}=\frac{\partial B_{z}}{\partial y}-\frac{\partial B_{y}}{\partial z}-\frac{1}{c^{2}} \frac{\partial}{\partial t}\left(E_{x}+v B_{y}-w B_{z}\right) \\
j_{y}=\frac{\partial B_{x}}{\partial z}-\frac{\partial B_{z}}{\partial x}-\frac{1}{c^{2}} \frac{\partial}{\partial t}\left(E_{y}+v B_{x}-u B_{z}\right) \\
j_{z}=\frac{\partial B_{y}}{\partial x}-\frac{\partial B_{x}}{\partial y}-\frac{1}{c^{2}} \frac{\partial}{\partial t}\left(E_{z}+u B_{y}-w B_{x}\right)
\end{gathered}
$$

$\varepsilon_{e, i}$ are the internal energies of the electronic and ionic components per unit mass, $W_{e, i}=\left(\left(W_{e, i}\right)_{x},\left(W_{e, i}\right)_{y},\left(W_{e, i}\right)_{z}\right)$ are the electron and ion heat fluxes, $c$ is speed of light in vacuum.

Heat fluxes are defined by ion and electron temperature gradients: 


$$
\begin{aligned}
& \left(W_{e, i}\right)_{x}=-\left(\kappa_{e, i}\right)_{x x} \frac{\partial T_{e, i}}{\partial x}-\left(\kappa_{e, i}\right)_{x y} \frac{\partial T_{e, i}}{\partial y}-\left(\kappa_{e, i}\right)_{x z} \frac{\partial T_{e, i}}{\partial z} \\
& \left(W_{e, i}\right)_{y}=-\left(\kappa_{e, i}\right)_{y x} \frac{\partial T_{e, i}}{\partial x}-\left(\kappa_{e, i}\right)_{y y} \frac{\partial T_{e, i}}{\partial y}-\left(\kappa_{e, i}\right)_{y z} \frac{\partial T_{e, i}}{\partial z} \\
& \left(W_{e, i}\right)_{z}=-\left(\kappa_{e, i}\right)_{z x} \frac{\partial T_{e, i}}{\partial x}-\left(\kappa_{e, i}\right)_{z y} \frac{\partial T_{e, i}}{\partial y}-\left(\kappa_{e, i}\right)_{z z} \frac{\partial T_{e, i}}{\partial z}
\end{aligned}
$$

where

$$
\begin{aligned}
& \left(\kappa_{e, i}\right)_{\xi \xi}=\left(\kappa_{e, i}\right)_{\|}\left(\frac{B_{\xi}}{B}\right)^{2}+\left(\kappa_{e, i}\right)_{\perp}\left(1-\left(\frac{B_{\xi}}{B}\right)\right)^{2}, \quad \xi=x, y, z \\
& \left(\kappa_{e, i}\right)_{\xi \zeta}=\left(\kappa_{e, i}\right)_{\zeta \xi}=\left[\left(\kappa_{e, i}\right)_{\|}-\left(\kappa_{e, i}\right)_{\perp}\right] \frac{B_{\xi} B_{\zeta}}{B^{2}}, \xi=x, y, z ; \zeta=x, y, z ; \xi \neq \zeta
\end{aligned}
$$

The equations of the electromagnetic field are applied in the following form:

$$
\begin{gathered}
\rho \frac{d}{d t}\left(\frac{B_{x}}{\rho}\right)=\frac{\partial E_{y}}{\partial z}-\frac{\partial E_{z}}{\partial y}+\left(B_{x} \frac{\partial u}{\partial x}+B_{y} \frac{\partial u}{\partial y}+B_{z} \frac{\partial u}{\partial z}\right) \\
\rho \frac{d}{d t}\left(\frac{B_{y}}{\rho}\right)=\frac{\partial E_{z}}{\partial x}-\frac{\partial E_{x}}{\partial z}+\left(B_{x} \frac{\partial w}{\partial x}+B_{y} \frac{\partial w}{\partial y}+B_{z} \frac{\partial w}{\partial z}\right), \\
\rho \frac{d}{d t}\left(\frac{B_{z}}{\rho}\right)=\frac{\partial E_{x}}{\partial y}-\frac{\partial E_{y}}{\partial x}+\left(B_{x} \frac{\partial v}{\partial x}+B_{y} \frac{\partial v}{\partial y}+B_{z} \frac{\partial v}{\partial z}\right), \\
E_{\xi}=\frac{1}{4 \pi_{\sigma_{\perp}}} j_{\xi}+\frac{\sigma_{\perp}-\sigma_{\|}}{4 \pi \sigma_{\perp} \sigma_{\|}} \frac{B_{\xi}}{B^{2}}(j, B) ; \quad \xi=x, y, z ;(j, B)=j_{x} B_{x}+j_{y} B_{y}+j_{z} B_{z} .
\end{gathered}
$$

The MHD system (1) - (9) is closed by the plasma equation of state (EOS).

To solve the MHD initial-boundary-value problem, the usual boundary conditions [13] are added to equations (1) - (9), which determine the hydrodynamic fluxes of mass, energy, and momentum, temperature or heat fluxes, and the conditions for the components of the electromagnetic field.

\section{COMPUTATIONAL ALGORITHM}

The difference model is built using staggered grid functions. Thermodynamic parameters (density, pressure, internal energy) as well as magnetic induction are defined in the grid cells. The components of the velocity and electric field strength are defined in the nodes. The difference equations of continuity, energy, momentum, and magnetic induction are derived by approximating the corresponding differential balance for control volume. The control volumes are either grid cells (for continuity and energy equations), or "nodal" volumes (for momentum balance equations), which form a grid conjugate to the original one. Timedependent pressure forces, ponderomotive forces, flows, etc. are included as a linear combination of the corresponding spatial approximations at two consecutive time layers. Weighting factors in time-weighted difference formulas were chosen to ensure complete 
conservativeness of the scheme. For the dissipative processes (thermal conductivity, field diffusion), homogeneous flux schemes were constructed. The theoretical foundations of such schemes are considered in [14] for the 2D case. Here we present a 3D version of the technique [14].

The numerical solution of the governing MHD system is performed according to the twostage algorithm which is analogues to that first proposed in [16] and used in many successive works. The movement of matter is calculated in the coordinate system $(\alpha, \beta, \gamma)$, which is moving relative to the laboratory $\operatorname{system}(x, y, z)$.

If $\mathbf{U}=(u, w, v)$ is the velocity of a material particle in the laboratory coordinate system, then $\tilde{\mathbf{U}}=\left(\tilde{U}_{x}, \tilde{U}_{y}, \tilde{U}_{z}\right)=\left.(u-d x / d t, w-d y / d t, v-d z / d t)\right|_{(\alpha, \beta, \gamma)=\text { const }}$ is its velocity in the moving system.

The time-advance begins by solving the main system of equations in Lagrange variables (i.e., without taking into account convective flows), then, if necessary, the Lagrangian grid is corrected, and the computed values are recalculated to a new difference grid.

The first (Lagrangian) stage includes solving the grid equations, provided that the grid moves at the speed of the substance. In this case, the Jacobian $\mathbf{J}$ changes according to the

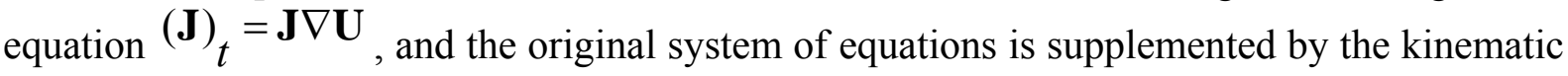
relation $\frac{\mathrm{d} \mathbf{r}(\alpha, \beta, \gamma, t)}{\mathrm{d} t}=\mathbf{U}(\mathbf{r})$ ( $\mathbf{r}$ is the radius vector of the material particle). At the end of the Lagrangian stage of calculating the position of the grid nodes, they can be redefined based on the solution strategy. In this case, the second (Eulerian) stage is performed that is the calculation of convective fluxes in the "grid" coordinate system and the consequent changes in the grid functions.

The above decomposition of the solution of the general system of equations into stages can be represented in the form of a summarized approximation scheme, or a physical splitting scheme. The advantage of the proposed algorithm is the possibility to use an implicit Lagrangian scheme and an explicit Eulerian scheme. This combination allows a larger time integration step without loss of stability.

The overall iterative cycle is based on the sequential computation of physical processes. It includes several nested cycles for the subsystems of the general system. The Lagrangian stage can be considered as iterations of the force and energy balances. At this stage the MHD system is solved coupled with the equations for the nodes coordinates moving in physical space with the speed of the matter. In turn, the Lagrangian stage itself is split into the ideal MHD (hyperbolic type subsystem) and the dissipative processes (parabolic type equations).

Lagrangian grids simulate the motion of liquid particles or liquid contours. This approach allows very simple and convenient form of the continuity equations, freezing of the field in the MHD equations, and lastly, an effective rapidly converging iterative algorithm for solving a system of grid equations corresponding to an implicit difference scheme. The convergence of the algorithm was studied in [14].

The Eulerian stage returns the nodes of the moving grid to their start-of-the-time-step positions. This is understood as the up-flow shift of the Lagrangian grid, due to which the substance moves through the edges of the cells. At this stage, changes in the grid functions ( $\rho$, $\mathbf{U}, \varepsilon, \mathbf{B})$ caused by convection processes are calculated. Note that the return of the grid to its 
previous position is optional, which allows lower numerical diffusion and improving the deformations of the moving grid.

The power and energy balances are interconnected. The plasma momentum $\rho \mathbf{U}(x, y, z, t)$ is determined by the pressure gradient and electromagnetic force dependent, in particular, on the temperature of the medium. In turn, the temperature field $T(x, y, z, t)$ depends on the work of the pressure forces and the energy flows, i.e. the plasma velocity. Therefore, the individual steps of solving the complete system of MHD grid equations are combined into an overall iterative cycle.

The system we solve includes difference equations of ideal MHD coupled with energy balance equations of the plasma components in a non-divergent (entropy) form and multigroup radiative transfer equations. The difference scheme of the Lagrangian stage is arranged in such a way that a difference analogue of the plasma - electromagnetic field integral energy balance is satisfied. This property is used to monitor the convergence of iterations at the Lagrangian stage and the quality of the solution in general.

The time derivatives of any function $f$ in the moving coordinate system are calculated according to the relation $\dot{f}=f_{t}+\mathbf{U} \nabla f$.

\section{COMPUTATIONAL GRID, DISCRETIZATION, DIFFERENCE EQUATIONS}

The approximation on a moving grid is implemented via mapping a single-connected region with a piecewise-smooth boundary $D \subset R^{3}(x, y, z)$ to the unit cube $D^{\prime} \subset R^{3}(\alpha, \beta, \gamma)[4,9$, 10]. In general the coordinate transformation formulas are time dependent. At any point of time the map is defined as:

$$
\begin{aligned}
& x=x(\alpha, \beta, \gamma), \quad y=y(\alpha, \beta, \gamma), \quad z=z(\alpha, \beta, \gamma) \\
& (x, y, z) \in D, \quad(\alpha, \beta, \gamma) \in D^{\prime}
\end{aligned}
$$

We suppose the positivity of the coordinate mapping Jacobian:

$$
\mathbf{J}=\frac{\partial(x, y, z)}{\partial(\alpha, \beta, \gamma)}>0,(\alpha, \beta, \gamma) \in D^{\prime}
$$

The volume of a cell in the physical space $(x, y, z)$ is approximated as the volume of its image in the reference space of the variables $(\alpha, \beta, \gamma)$ multiplied by the average value of the Jacobian $\langle\mathbf{J}\rangle$ in the cell.

\subsection{System of difference equations}

The MHD system (1) - (9) is approximated on a moving (Lagrangian) difference grid by a completely conservative difference scheme.

The corresponding numerical model is as follows.

A uniform in each direction grid is introduced in the cube $D^{\prime}(\alpha, \beta, \gamma)$ : 


$$
\begin{aligned}
& \alpha_{i}=i h_{\alpha}, i=\overline{0, N M}, h_{\alpha}=1 / N M, \\
\omega_{h}: \quad & \beta_{j}=j h_{\beta}, j=\overline{0, N L}, h_{\beta}=1 / N L, \\
& \gamma_{k}=k h_{\gamma}, k=\overline{0, N N}, h_{\gamma}=1 / N N .
\end{aligned}
$$

We denote by $\omega$ the set of cells of the computational grid $\omega_{h}, \Omega$ is the set of nodes, $\theta$ is the set of faces. Accordingly, we introduce the spaces of grid functions defined in the cells $H_{\omega}$, in the nodes $H_{\Omega}$, and in the faces $H_{\theta}$. We use the indices $(i, j, k)$ for the grid functions $f \in H_{\Omega}$ : $f_{i, j, k}=f \in H_{\Omega}$, and indices $(m, l, n)$ for the grid functions $\varphi \in H_{\omega}: \varphi_{\min } \in H_{\omega}$. The functions $\psi \in H_{\theta}$ will be marked with indices $(i, l, n),(m, j, n)$ and $(m, l, k)$.

The grid in the region $D$ obtained by the mapping (10) consists of hexagons.

The difference equations below use grid templates $\Xi_{1}, \Xi_{2}, \Xi_{3}$ :

- $\Xi_{l}$ is the cell template, it includes the incident nodes;

- $\Xi_{2}$ is the node template, it includes the incident cells;

- $\Xi_{3}$ is the pattern of faces incident to the cell $(m, l, n)$.

Equations (1) - (9) are approximated on the grid $H_{\omega}$ by a completely conservative implicit difference scheme. The difference balance equations for the mass, momentum, and energy in a control volume are constructed using the partial derivatives of the cell volumes with respect to the node coordinates (for theoretical grounds see $[17,26]$ ):

$$
\begin{aligned}
& x_{t}=u^{(0.5)} ; y_{t}=w^{(0.5)} ; z_{t}=v^{(0.5)} \\
& \Delta m=\rho\langle\mathbf{J}\rangle h_{\alpha} h_{\beta} h_{\gamma}=\rho V=\hat{\rho} \hat{V}, \\
& P=P_{e}+P_{i}+q \\
& M u_{t}=\sum_{r \in \Xi_{2}}\left(\frac{\partial \widehat{V}_{r}}{\partial x}\right)\left(\widehat{P}_{r}+\frac{B_{r} \widehat{B}_{r}}{8 \pi}\right)-\frac{1}{4 \pi} \sum_{r \in \Xi_{2}} B_{x_{r}}^{(0.5)} \partial B_{x} \\
& M w_{t}=\sum_{r \in \Xi_{2}}\left(\frac{\partial \widehat{V}_{r}}{\partial y}\right)\left(\widehat{P}_{r}+\frac{B_{r} \widehat{B}_{r}}{8 \pi}\right)-\frac{1}{4 \pi} \sum_{r \in \Xi_{2}} B_{y_{r}}^{(0.5)} \partial B_{y} \\
& M v_{t}=\sum_{r \in \Xi_{2}}\left(\frac{\partial \hat{V}_{r}}{\partial z}\right)\left(\hat{P}_{r}+\frac{B_{r} \widehat{B}_{r}}{8 \pi}\right)-\frac{1}{4 \pi} \sum_{r \in \Xi_{2}} B_{z_{r}}^{(0.5)} \partial B_{z} \\
& \partial B_{\xi}=\sum_{r \subset \Xi_{1}} \frac{\partial \widehat{V}_{r}}{\partial \xi} \widehat{B}_{\xi_{r}}, \quad \xi=x, y, z, \quad M=\frac{1}{8} \sum_{r \in \Xi_{2}} \Delta_{m_{r}} \\
& \left(V B_{x}\right)_{t}=\sum_{\xi=x, y, z} \widehat{B}_{\xi} \sum_{r \in \Xi_{1}} \frac{\partial \hat{V}}{\partial \alpha_{r}} u_{r}^{(0.5)}+\sum_{r \in \Xi_{1}}\left[\left(\frac{\partial \hat{V}}{\partial z_{r}}\right) \widehat{E}_{y_{r}}-\left(\frac{\partial \hat{V}}{\partial y_{r}}\right) \widehat{E}_{z_{r}}\right] \\
& \left(V B_{y}\right)_{t}=\sum_{\xi=x, y, z} \widehat{B}_{\xi} \sum_{r \in \Xi_{1}} \frac{\partial \hat{V}}{\partial \alpha_{r}} w_{r}^{(0.5)}+\sum_{r \in \Xi_{1}}\left[\left(\frac{\partial \hat{V}}{\partial x_{r}}\right) \widehat{E}_{z_{r}}-\left(\frac{\partial \hat{V}}{\partial z_{r}}\right) \widehat{E}_{x_{r}}\right] \\
& \left(V B_{z}\right)_{t}=\sum_{\xi=x, y, z} \widehat{B}_{\xi} \sum_{r \in \Xi_{1}} \frac{\partial \hat{V}}{\partial \alpha_{r}} v_{r}^{(0.5)}+\sum_{r \in \Xi_{1}}\left[\left(\frac{\partial \hat{V}}{\partial y_{r}}\right) \widehat{E}_{x_{r}}-\left(\frac{\partial \hat{V}}{\partial x_{r}}\right) \widehat{E}_{y_{r}}\right]
\end{aligned}
$$




$$
\begin{aligned}
& \widehat{E}_{\xi}=\frac{1}{4 \pi \hat{\sigma}_{\perp}} \hat{j}_{\xi}+\frac{\hat{\sigma}_{\perp}-\hat{\sigma}_{\|}}{4 \pi_{\hat{\sigma}_{\perp} \hat{\sigma}_{\|}}} \frac{\hat{B}_{\xi}}{\hat{B}^{2}} B^{* * *}, \quad \xi=x, y, z \\
& B^{* * *}=\widehat{B}_{x} \tilde{j}_{x}+\widehat{B}_{y} \tilde{j}_{y}+\widehat{B}_{z} \tilde{j}_{z} \\
& \tilde{j}_{x}=-\frac{1}{\widehat{V}} \sum_{r \in \Xi_{2}}\left[\frac{\partial V_{r}}{\partial y} \widehat{B}_{z_{r}}-\frac{\partial V_{r}}{\partial z} \widehat{B}_{y_{r}}\right]-\frac{1}{c^{2}}\left(E_{x}+v B_{y}-w B_{z}\right)_{t} \\
& \tilde{j}_{y}=-\frac{1}{\widehat{V}} \sum_{r \in \Xi_{2}}\left[\frac{\partial V_{r}}{\partial z} \widehat{B}_{x_{r}}-\frac{\partial V_{r}}{\partial x} \widehat{B}_{z_{r}}\right]-\frac{1}{c^{2}}\left(E_{y}+w B_{z}-u B_{x}\right)_{t} \\
& \tilde{j}_{z}=-\frac{1}{\widehat{V}} \sum_{r \Xi_{2}}\left[\frac{\partial V_{r}}{\partial x} \widehat{B}_{y_{r}}-\frac{\partial V_{r}}{\partial y} \widehat{B}_{r}\right]-\frac{1}{c^{2}}\left(E_{z}+u B_{x}-v B_{y}\right)_{t} \\
& \left(\varepsilon_{e}\right)_{t}=-\bar{P}_{e}\left(\frac{1}{\rho}\right)_{t}+\widehat{Q}_{e i}+\widehat{G}_{e}+\frac{1}{\Delta m} \sum_{r \in \Xi_{3}} \sum_{\xi=x, y, z} \widehat{W}_{e, \xi r} \hat{S}_{\xi r}+\widehat{G}_{j} \\
& \left(\varepsilon_{i}\right)_{t}=-\left(\widehat{P}_{i}+\hat{q}\right)\left(\frac{1}{\rho}\right)_{t}-\widehat{Q}_{e i}+\frac{1}{\Delta m} \sum_{r \in \Xi_{3}} \sum_{\xi=x, y, z} \widehat{W}_{i, \xi r} \widehat{S}_{\xi r}+\widehat{G}_{i}
\end{aligned}
$$

Joule heating:

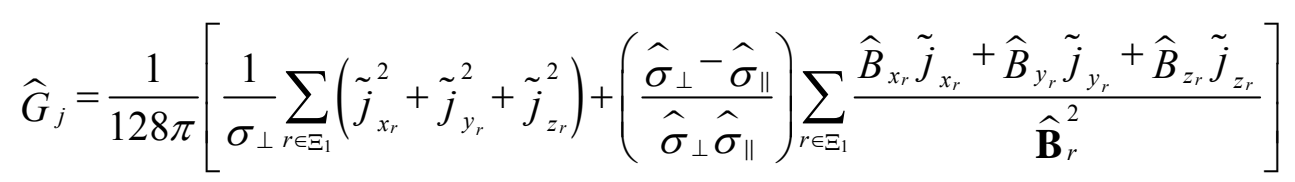

We use index-free notation to represent the system (11)-(18) in a compact form [1].

The grid functions $\mathbf{U}, \mathbf{E}$ are defined in the nodes of the difference grid, the grid functions $\rho$, $P_{e, i}, q, V, T_{e, i}, \mathbf{B}, G_{e, i}, Q_{e i}, \kappa_{e, i}, \sigma$ are defined in the cells ( $q$ is the volumetric artificial viscosity; $V$ is the volume of the cell), and the grid functions $\mathbf{W}_{e, i}, \mathbf{S}$ are defined on the faces of the cells. $\mathbf{S}_{r}=\left(S_{x r}, S_{y r} S_{z r}\right), r=\overline{1,6}$ are the areas of the faces of the cell $(m, l, n)$.

The boundary values of pressure, temperature, and magnetic field are assigned to the faces of the boundary cells.

At the boundary of the computational region, for the equations of motion, the pressure and velocity, or the absence of a plasma flow condition is specified. For Maxwell's equations, the magnetic induction or the electric field strength is specified. For energy equations, the distribution of temperature or heat fluxes is specified at the boundaries.

\subsection{Energy balance and conservation}

The difference scheme (10) - (17) is completely conservative. We define the following grid functionals to prove this property and to utilize it in practical calculations:

$$
\begin{aligned}
& \varepsilon_{k i n}=\frac{1}{2} \sum_{m=1}^{N M} \sum_{l=1}^{N L} \sum_{n=1}^{N N} \Delta m_{m l n}\left(u_{m l n}^{2}+w_{m l n}^{2}+v_{m l n}^{2}\right) \\
& u_{m l n}=\frac{1}{8} \sum_{r \in \Xi_{1}} u_{r}, \quad w_{m l n}=\frac{1}{8} \sum_{r \in \Xi_{1}} w_{r}, \quad v_{m l n}=\frac{1}{8} \sum_{r \in \Xi_{1}} v_{r}
\end{aligned}
$$




$$
\begin{aligned}
& E_{\text {tot }}=\sum_{m l n} \Delta m_{m l n}\left(\varepsilon_{e_{m l n}}+\varepsilon_{i_{m l n}}+\frac{u_{m l n}^{2}+w_{m l n}^{2}+v_{m l n}^{2}}{2}+\frac{B_{x_{m l n}}^{2}+B_{y_{m l n}}^{2}+B_{z_{m l n}}^{2}}{8 \pi \rho_{m l n}}\right) \\
& u_{m l n}^{2}=\frac{1}{8} \sum_{r \in \Xi_{1}} u_{r}^{2}, \quad w_{m l n}^{2}=\frac{1}{8} \sum_{r \in \Xi_{1}} w_{r}^{2}, \quad v_{m l n}^{2}=\frac{1}{8} \sum_{r \in \Xi_{1}} v_{r}^{2} .
\end{aligned}
$$

The following indices are used for energy balance relations:

$(i, j, k)$ for boundary nodes, $r^{\prime}$ for cells adjacent to the boundary, $r$ " for "ghost" cells introduced into difference scheme for processing of boundary conditions:

$$
\begin{gathered}
\sum_{r \in \Xi_{2}}=\sum_{r^{\prime} \in \Xi_{2}}+\sum_{r^{\prime \prime} \in \Xi_{2}} \\
\mu_{i j k}^{\prime}=\frac{1}{8 M_{i j k}} \sum_{r^{\prime} \in \Xi_{2}} \Delta m_{r^{\prime}}, \mu_{i j k}^{\prime \prime}=\frac{1}{8 M_{i j k}} \sum_{r^{\prime \prime} \in \Xi_{2}} \Delta m_{r^{\prime \prime}}=1-\mu_{i j k}^{\prime} \\
\eta_{i j k}^{\prime}=\frac{1}{8 \hat{V}_{i j k}} \sum_{r^{\prime} \in \Xi_{2}} \hat{V}_{r^{\prime}}, \eta_{i j k}^{\prime \prime}=1-\eta_{i j k}^{\prime}
\end{gathered}
$$

The equation of total energy balance for the coupled plasma - electromagnetic field system (22) that follows from the system of difference equations (10) - (17) can be found in Appendix.

From this we can conclude that for the difference model $(11)-(18)$, the change in the total energy is determined by:

1. The work of external forces, i.e. pressure and ponderomotive force;

2. The influx (outflow) of heat trough the border of the region, including radiative heat transfer;

3. The influx of magnetic energy through the outer boundary;

4. The action of energy sources and sinks.

\subsection{Combined iterative method for the system of difference equations}

The difference equations (11) - (18) represent a system of nonlinear algebraic equations. An iterative method is used with separate solving groups of equations for different physical processes.

The procedure for solving system (11) - (18) is as follows:

1. The auxiliary values of velocity, density, temperature, and magnetic induction at the time layer $(n+1)$ are computed.

2. With the fixed temperature, the equations of motion and the Maxwell equations are solved. The auxiliary values of the velocity, density, and electromagnetic field parameters are computed.

3. With the fixed velocity, density and electromagnetic field, the system of energy equations is solved. The auxiliary values of the electron and ion temperature at the time layer $(n+1)$ are computed. The equation of state is a link between the equations of the "first group" (equations of dynamics and electromagnetic field) and the "second group" (energy balance equation).

4. The satisfaction of the energy conservation law (22) is verified. If the required accuracy is achieved, the values of the functions at time $t=(n+1)$ are considered to be found. 
Otherwise we correct the coefficient of the system and repeat the step 1 to 3 .

5. If necessary, the grid is corrected after solving the system (11)-(18). The grid functions found at the Lagrangian stage are recalculated to the adjusted grid. For this, the conversion algorithm developed in $[5,6]$ is used.

The steps 2 and 3 are detailed below.

\subsection{Coupled dynamics and electrodynamics equations}

At the step 2, the subsystem of difference equations (11) - (16) is split by physical processes: first, the motion of a substance is calculated under the frozen magnetic field assumption, and then the diffusion of the magnetic field is accounted. The equations are solved by the Newton method with the reduction of unknown quantities [4]. The convergence of this procedure is considered, e.g., in [18]. Advancing from iteration $(s)$ to iteration $(s+1)$, we assume that all the variables in the equations of motion depend only on the velocity components $u_{i j k}, w_{i j k}, v_{i j k}$, and the thermal pressure is locally barotropic. The transition formulae from iteration $(s)$ to iteration $(s+1)$ are the following:

$$
\begin{aligned}
& u_{i j k}^{s+1}=u_{i j k}^{s}+\delta u_{i j k} \\
& w_{i j k}^{s+1}=w_{i j k}^{s}+\delta w_{i j k} \\
& v_{i j k}^{s+1}=v_{i j k}^{s}+\delta v_{i j k} \\
& x_{i j k}^{s+1}=x_{i j k}^{s}+\delta x_{i j k}=x_{i j k}^{s}+\frac{\partial x_{i j k}}{\partial u_{i j k}} \delta u_{i j k}=x_{i j k}^{s}+\frac{\Delta t}{2} \delta u_{i j k} \\
& y_{i j k}^{s+1}=y_{i j k}^{s}+\delta y_{i j k}=y_{i j k}^{s}+\frac{\partial y_{i j k}}{\partial w_{i j k}} \delta_{w_{i j k}}=y_{i j k}^{s}+\frac{\Delta t}{2} \delta_{w_{i j k}} \\
& z_{i j k}^{s+1}=z_{i j k}^{s}+\delta z_{i j k}=z_{i j k}^{s}+\frac{\partial z_{i j k}}{\partial v_{i j k}} \delta_{v_{i j k}}=z_{i j k}^{s}+\frac{1}{2} \delta_{v_{i j k}} \\
& \Phi_{m l n}^{s+1}=\Phi_{m l n}^{s}+\delta \Phi_{m l n}=\Phi_{m l n}^{s}+\sum_{r \in \Xi_{1}}\left(\frac{\partial \Phi_{m l n}}{\partial u_{r}} \delta_{u_{r}}+\frac{\partial \Phi_{m l n}}{\partial w_{r}} \delta_{w_{r}}+\frac{\partial \Phi_{m l n}}{\partial v_{r}} \delta_{v_{r}}\right)
\end{aligned}
$$

Here $\Phi_{m l n}$ is for cell functions $P_{m l n}, q_{m l n}, B_{m l n}$, and superscripts are for the iteration number.

Substituting the values (23) into the equations (14) and neglecting the squares of the increments of the functions, we obtain a system of linear algebraic equations with respect to the increments of the velocity components $\delta u_{i j k}, \delta w_{i j k}, \delta v_{i j k}$ :

$$
\begin{aligned}
& \sum_{k_{1}=-1}^{1} \sum_{k_{2}=-1}^{1} \sum_{k_{3}=-1}^{1}\left(a_{1 j i k}^{\left(k_{1} k_{2} k_{3}\right)} \delta_{u_{i+k_{1} j+k_{2} k+k_{3}}}+b_{1 j i k}^{\left(k_{1} k_{2} k_{3}\right)} \delta_{W_{i+k_{1} j+k_{2} k+k_{3}}}+c_{1 j j k}^{\left(k_{1} k_{2} k_{3}\right)} \delta_{v_{i+k_{1} j+k_{2} k+k_{3}}}\right)=F_{i j k}^{(1)} \\
& \sum_{k_{1}=-1}^{1} \sum_{k_{2}=-1}^{1} \sum_{k_{3}=-1}^{1}\left(a_{2 i j k}^{\left(k_{1} k_{2} k_{3}\right)} \delta u_{i+k_{1} j+k_{2} k+k_{3}}+b_{2 i j k}^{\left(k_{1} k_{2} k_{3}\right)} \delta w_{i+k_{1} j+k_{2} k+k_{3}}+c_{2 i j k}^{\left(k_{1} k_{2} k_{3}\right)} \delta v_{i+k_{1} j+k_{2} k+k_{3}}\right)=F_{i j k}^{(2)} \\
& \sum_{k_{1}=-1}^{1} \sum_{k_{2}=-1}^{1} \sum_{k_{3}=-1}^{1}\left(a_{3 i j k}^{\left(k_{1} k_{2} k_{3}\right)} \delta u_{i+k_{1} j+k_{2} k+k_{3}}+b_{3 i j k}^{\left(k_{1} k_{2} k_{3}\right)} \delta_{w_{i+k_{1} j+k_{2} k+k_{3}}}+c_{3 i j k}^{\left(k_{1} k_{2} k_{3}\right)} \delta_{v_{i+k_{1} j+k_{2} k+k_{3}}}\right)=F_{i j k}^{(3)} \\
& i=\overline{1, N M}, j=\overline{1, N L}, k=\overline{1, N N}
\end{aligned}
$$


Here $a_{1,2,3 i j k}^{\left(k_{1} k_{2} k_{3}\right)}, b_{1,2,3 i j k}^{\left(k_{1} k_{2} k_{3}\right)}, c_{1,2,3 i j k}^{\left(k_{1} k_{2} k_{3}\right)}, F_{i j k}^{(1,2,3)}$ are numeric factors. System of equations (24) has a block structure matrix.

To solve equations (24), we use iterative method [29].

After finding the velocity increment at the iteration $(s+1)$, the values $\delta u_{i j k}, \delta w_{i j k}, \delta v_{i j k}, V_{m l n}$, $\rho_{m l n}, P_{m l n}, q_{m l n}$ are computed, and the intermediate value of the magnetic induction $\tilde{\mathbf{B}}$ :

$$
\tilde{\mathbf{B}}_{m l n}=\mathbf{B}\left(\rho_{m l n}^{s+1}, \mathbf{E}^{s}\right)
$$

The next step is to solve the system of equations describing the electric and magnetic fields with finite conductivity of the medium $(15)-(16)$. The intermediate value $\tilde{\mathbf{B}}$ is used for calculating the conductivity coefficient:

$$
\sigma_{m l n}^{s+1}=\sigma_{m l n}\left(\rho_{m l n}^{s+1}, \tilde{\mathbf{B}}_{m l n}, T_{m l n}^{s}\right)
$$

Then the system of difference equations with respect to $\mathbf{B}^{s+1}, \mathbf{E}^{s+1}$ is linear. Because some rarefied plasma areas may have close to zero conductivity, it is advisable to solve the system of field equations by excluding the magnetic induction $\mathbf{B}$. The result is a system of linear equations with respect to $E_{x}, E_{y}, E_{z}$ :

$$
\begin{aligned}
& \sum_{k_{1}=-1}^{1} \sum_{k_{2}=-1}^{1} \sum_{k_{3}=-1}^{1}\left(\bar{a}_{1 i j k}^{\left(k_{1} k_{2} k_{3}\right)}\left(\widehat{E}_{x}\right)_{i+k_{1} j+k_{2} k+k_{3}}+\bar{b}_{1 i j k}^{\left(k_{1} k_{2} k_{3}\right)}\left(\widehat{E}_{y}\right)_{i+k_{1} j+k_{2} k+k_{3}}+\bar{c}^{\left(k_{1 j} k_{2} k_{3}\right)}\left(\widehat{E}_{z}\right)_{i+k_{1} j+k_{2} k+k_{3}}\right)=\bar{F}_{i j k}^{(1)} \\
& \sum_{k_{1}=-1}^{1} \sum_{k_{2}=-1}^{1} \sum_{k_{3}=-1}^{1}\left(\bar{a}_{2 i j k}^{\left(k_{1} k_{2} k_{3}\right)}\left(\widehat{E}_{x}\right)_{i+k_{1} j+k_{2} k+k_{3}}+\bar{b}_{2 i j k}^{\left(k_{1} k_{k} k_{3}\right)}\left(\widehat{E}_{y}\right)_{i+k_{1} j+k_{2} k+k_{3}}+\bar{c}_{2 i j k}^{\left(k_{1} k_{2} k_{3}\right)}\left(\widehat{E}_{z}\right)_{i+k_{1} j+k_{2} k+k_{3}}\right)=\bar{F}_{i j k}^{(2)} \\
& \sum_{k_{1}=-1}^{1} \sum_{k_{2}=-1}^{1} \sum_{k_{3}=-1}^{1}\left(a_{3 i j k}^{\left(k_{1} k_{2} k_{3}\right)}\left(\widehat{E}_{x}\right)_{i+k_{1} j+k_{2} k+k_{3}}+b_{3 i j k}^{\left(k_{1} k_{2} k_{3}\right)}\left(\widehat{E}_{y}\right)_{i+k_{1} j+k_{2} k+k_{3}}+c_{3 i j k}^{\left(k_{1} k_{2} k_{3}\right)}\left(\widehat{E}_{z}\right)_{i+k_{1} j+k_{2} k+k_{3}}\right)=\bar{F}_{i j k}^{(3)} \\
& i=\overline{1, N M}, j=\overline{1, N L}, k=\overline{1, N N} \\
& \text { Here } \bar{a}_{1,2,3 i j k}^{\left(k_{1} k_{2} k_{3}\right)}, \bar{b}_{1,2,3 i j k}^{\left(k_{1} k_{2} k_{3}\right)}, \bar{c}_{1,2,3 i j k}^{\left(k_{1} k_{2} k_{3}\right)}, \bar{F}_{i j k}^{(1,2,3)} \text { are numeric factors. }
\end{aligned}
$$

The system of linear equations (25) has a block structure. After finding the electric fields, we have:

$$
\mathbf{B}_{m l n}^{s+1}=\mathbf{B}\left(\rho_{m l n}^{s+1}, \mathbf{E}^{s+1}\right)
$$

The system of equations (11) - (16) is solved if the increments of velocities at the iteration satisfy the conditions:

$$
\begin{aligned}
& \left|\delta u_{i j k}\right| \leq \varepsilon_{\mu}\left|u_{i j k}\right|+u_{\min } \\
& \left|\delta w_{i j k}\right| \leq \varepsilon_{\mu}\left|w_{i j k}\right|+w_{\min } \\
& \left|\delta v_{i j k}\right| \leq \varepsilon_{\mu}\left|v_{i j k}\right|+v_{\min }
\end{aligned}
$$

Here $\varepsilon_{\mu}$ is the relative velocity error, and $u_{\min }, v_{\min }, w_{\min }$ are the absolute velocity errors.

\subsection{Energy Balance}

The energy balance equations are solved in terms of electron and ion temperature (17). 
Energy equations are solved via the Newton iterations. The transition formulae from iteration $(s)$ to iteration $(s+1)$ are the following:

$$
\begin{aligned}
& \left(T_{e, i}\right)_{m l n}^{s+1}=\left(T_{e, i}\right)_{m l n}^{s}+\delta\left(T_{e, i}\right)_{m l n} \\
& \left(P_{e, i}\right)_{m l n}^{s+1}=\left(P_{e, i}\right)_{m l n}^{s}+\delta\left(P_{e, i}\right)_{m l n}=\left(P_{e, i}\right)_{m l n}^{s}+\left(\frac{\partial P_{e, i}}{\partial T_{e, i}}\right)_{m l n} \delta\left(T_{e, i}\right)_{m l n} \\
& \left(Q_{e i}\right)_{m l n}^{s+1}=\left(Q_{e i}\right)_{m l n}^{s}+\left(\frac{\partial Q_{e i}}{\partial T_{e}}\right)_{m l n} \delta\left(T_{e}\right)_{m l n}+\left(\frac{\partial Q_{e i}}{\partial T_{i}}\right)_{m l n} \delta\left(T_{i}\right)_{m l n} \\
& \left(\kappa_{e, i}\right)_{g}^{s+1}=\left(\kappa_{e, i}\right)_{g}^{s}+\sum_{r \in \Xi_{4}} \frac{\left(\partial \kappa_{e, i}\right)_{g}}{\left(\partial T_{e, i}\right)_{r}} \partial\left(T_{e, i}\right)_{r}
\end{aligned}
$$

$\Xi_{4}$ is a template of the cells adjacent to the face $g$.

Substituting increments of functions at the iteration $(s+1)$ into the energy equations and neglecting the squares of the increments, we obtain a system of linear algebraic equations with respect to the temperature increments $\delta\left(T_{e}\right)_{m l n}, \delta\left(T_{i}\right)_{m l n}$ :

$$
\begin{aligned}
& \sum_{k_{1}=-1}^{1} \sum_{k_{2}=-1}^{1} \sum_{k_{3}=-1}^{1}\left(\operatorname{a}_{m l n}^{\left(k_{1} k_{2} k_{3}\right)}\left(\delta T_{e}\right)_{m+k_{1} l+k_{2} n+k_{3}}\right)+\overline{\bar{b}}_{m l n}^{(0,0,0)}\left(\delta T_{i}\right)_{m l n}=\overline{\bar{F}}_{m l n}^{(1)} \\
& { }^{(0,0,0)}\left(\delta T_{i}\right)_{m l n}+\sum_{k_{1}=-1}^{1} \sum_{k_{2}=-1}^{1} \sum_{k_{3}=-1}^{1}\left(\overline{\bar{d}}_{m l n}^{\left(k_{1} k_{2} k_{3}\right)}\left(\delta T_{i}\right)_{m+k_{1} l+k_{2} n+k_{3}}\right)=\overline{\bar{F}}_{m l n}^{(2)}
\end{aligned}
$$

Here ${\stackrel{=}{a_{m l n}}}_{\left(k_{1} k_{2} k_{3}\right)}, \bar{b}_{m l n}^{(0,0,0)},{ }_{c_{m l n}}^{=(0,0,0)}, \overline{\bar{d}}_{m l n}^{\left(k_{1} k_{2} k_{3}\right)}, \overline{\bar{F}}_{m l n}^{(1,2)}$ are the coefficients obtained in the linearization procedure.

Similar equations at the boundary are obtained in accordance with the type of boundary conditions. For example, with a fixed temperature, all the temperature increments at the boundary are set equal to 0 .

The system of difference equations (28) is solved similarly to systems (24) and (25).

The energy equations are solved if the temperature increments satisfy the conditions

$$
\left|\delta\left(T_{e / i}\right)_{m l n}\right| \leq \varepsilon_{T}\left(T_{e / i}\right)_{m l n}+T_{\min }, m=\overline{1, N M-1}, l=\overline{1, N L-1}, n=\overline{1, N N-1}
$$

Here $\varepsilon_{T}$ and $T_{\min }$ are the relative and absolute temperature errors.

\section{SIMULATION OF A Z - PINCH IMPLOSION DYNAMICS}

The described technique was applied to simulations of $\mathrm{Z}$ - pinch plasmas experiments with the use of pulsed-power facilities. Three-dimensional modeling was carried out by means of the RMHD code MARPLE-3D [22]. We have studied the $\mathrm{Z}$ - pinch produced by a multiwire array heated in a powerful electric discharge. The aim of simulations was to assess the current-carrying plasma instabilities that occur at the final stage of pinch formation and their development up to the final stage of compression of the plasma compression. The spatial perturbations of matter and magnetic flux distribution inside the wire array and their evolution at various stages of pinch compression were investigated. 
The simulation results are compared with the experimental data obtained at the Angara-5-1 facility (Troitsk Institute for Innovative and Thermonuclear Investigations - TRINITI, Moscow, Russia). The calculations were performed for multiwire configurations described, e.g., in [24]. Multiwire arrays proved to be a very effective electric load due to possibility of flexible adjustment of its parameters to that of a pulsed-power electric generator. However, as a wire-array has inhomogeneous structure, the resulting Z-pinch is subjected to MHD instabilities.

The magnetic flux breakthrough into various multiwire arrays (tungsten, molybdenum, copper, and aluminum) during their implosion was studied experimentally at the Angara-5-1 facility [25]. It is shown that breakthroughs develop in the final stage of plasma production from the wires and occur near the initial wire position. The spatial distribution of the azimuthal magnetic field $B{ }_{\varphi}(z, t)$ was measured using magnetic probes. The characteristic dimensions of the regions with a nonuniform magnetic field at the outer boundary of the wire array plasma were determined and compared with those of the regions with depressed plasma radiation observed in frame and time-integrated X-ray images. The dynamics of the nonuniform magnetic field was compared with the pinch radiation at different stages of implosion exposed in the frame X-ray images. The plasma density in the magnetic flux breakthrough area was estimated.

The magnetic breakthrough phenomena is illustrated by the Fig.1. The experiment No. 5265 with a 40 aluminum wires array is typical for Z-pinch studies at ANGARA-5-1.
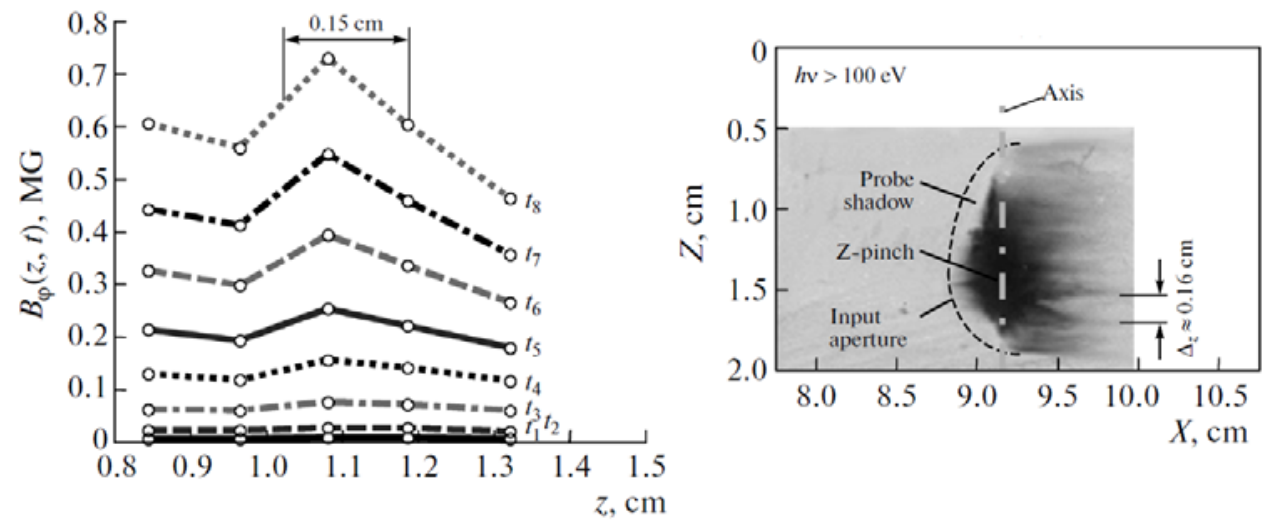

Figure 1. Experimental results (TRINITI, shot No. 5265 [25]).

At the left is the axial distributions of the azimuthal magnetic field inside the wire array measured at different instants of time $\left(t_{1}=30 \mathrm{~ns}, \ldots t_{7}=90 \mathrm{~ns}, t_{8}=110 \mathrm{~ns}\right)$ by the probe installed at $r_{p}=0.89 \mathrm{~cm}$. The coordinates $\mathrm{z}=0$ and $\mathrm{z}=1.4 \mathrm{~cm}$ correspond to the cathode and anode, respectively. At the right is the time integrated pinhole image (negative) of the wire array plasma recorded behind a Mylar film ( $\mathrm{h} v>100 \mathrm{eV}$ ). To the left of the axis, the image is absent because of the diaphragming of the input aperture of the pinhole camera. The anode is on the top, and the cathode is on the bottom.

The modeling was carried out by means of 3D RMHD code MARPLE, developed in KIAM RAS [22]. MARPLE is a full-scale multiphysics research code using the state-of-theart physics and numeric techniques. MARPLE provides a platform for high performance computing and functionality for solving the initial-boundary value problems using 
unstructured computational meshes. MARPLE physics includes: one-fluid two-temperature MHD model with electron-ion energy relaxation; general Ohm's law; anisotropic resistivity and heat conductivity in the magnetic field; radiative energy transfer (diffusion model, multigroup spectral model); multi-component convection-diffusion; wide-range equations of state (EOS), transport and kinetic coefficients, opacity and emissivity [23]. MARPLE main numerics are: mixed unstructured / block meshes (tetrahedral, hexahedral, prismatic elements and their combinations); high-resolution explicit TVD approximations to the ideal MHD equations; implicit FV/FE/DG techniques for dissipative processes; splitting scheme for RMHD system (elemental solvers for different physical processes, additive approximation scheme, conservation laws); 2-nd order predictor-corrector time-advance scheme. MARPLE is designed for high performance distributed computations using domain decomposition and MPI parallelism. The computing environment includes a set of service functions: data IO; mesh processing; parallel computations support; dynamic processing of computation objects (solvers, approximations, boundary conditions, matter properties); configurable recovery points writing and automated backup; advanced events logging. We use the open-source products: CAD-CAE platform SALOME [30] for complex computational domains (geometry description, setting boundary and subregions attributes, mesh generation and refinement), and multi-platform data analysis and visualization application ParaView [31].

The purpose of the simulation was to study the plasma instabilities at the final stage of imploded plasma stagnation. We present here the results of a plasma implosion simulation in accordance with the conditions of the experiment No. 5265. We studied a $20 \mathrm{~mm}$ diameter $14 \mathrm{~mm}$ high array made of $4015 \mu \mathrm{m}$ diameter Al wires with a total linear mass of $220 \mu \mathrm{g} / \mathrm{cm}$.

The computational domain was a cylindrical sector $45^{\circ}$ with periodic boundary conditions at $\varphi=0$ and $\varphi=\pi / 4$ (1/8 of the discharge chamber volume with 5 wires). The sector height was $3 \mathrm{~mm}(\sim 1 / 5$ of the array height). See Fig. 2, left. The grid contained 1.2 million cells (hexahedra and prisms). The grid in the $(x, y)$ plane was refined from $h_{x}=h_{y} \approx 80 \mu \mathrm{m}$ near the initial position of the wires to $h_{x}=h_{y} \approx 17 \mu \mathrm{m}$ near the axis, the grid along the $z$ axis was uniform, $h_{z}=30 \mu \mathrm{m}$. The electrodes were considered ideally conducting. At the outer wall of the discharge chamber, the boundary condition was set for the magnetic induction $B_{\varphi}=2 I / R$, where $R$ is the external radius of the discharge chamber, $I=I(t)$ is the total generator current through the array (experimental data, see Fig. 4).

Plasma emission from exploded wires was simulated using the model of prolonged plasma creation [21]. The rate of plasma production was calculated by the formula

$$
\dot{m}(t)= \begin{cases}k B(t)^{2}, & t<t_{\alpha}, \\ \frac{k B\left(t_{\alpha}\right)^{2}}{M_{0}(1-\alpha)}\left(M_{0}-m(t)\right), & t \geq t_{\alpha} .\end{cases}
$$

Here $m$ is the ablated mass, $M_{0}$ is the total mass of the array, $t_{\alpha}$ is defined from the condition $m\left(t_{\alpha}\right)=\alpha M_{0}$. The coefficient $k=2$ was chosen in accordance with the experimental data, so that the wire ablation ended approximately 10ns before the current maximum.

Spatial modulation of the plasma formation rate was introduced in accordance with the experimental data [25] by the formula

$$
\alpha=0,9(1-0,45[1+\sin (2 \pi z / \lambda)]), 0 \leq z \leq z_{\max },
$$


which corresponds to the experimentally observed electrical explosion inhomogeneities with a characteristic wavelength $\lambda \sim 100 \mu \mathrm{m}$.

The data tables of opacities and matter properties (equation of state) for the aluminum plasma were previously calculated using the TERMOS code developed in KIAM RAS [32].

A volumetric artificial (mathematical) viscosity was introduced into the difference scheme to ensure calculation of flows with strong radiative shocks. The viscosity value was regulated according the recommendations [15].

The calculations were performed on the supercomputers MVS-100K (JSCC RAS) and K100 (KIAM RAS). A typical run using 240 computing cores required up to 70 hours.
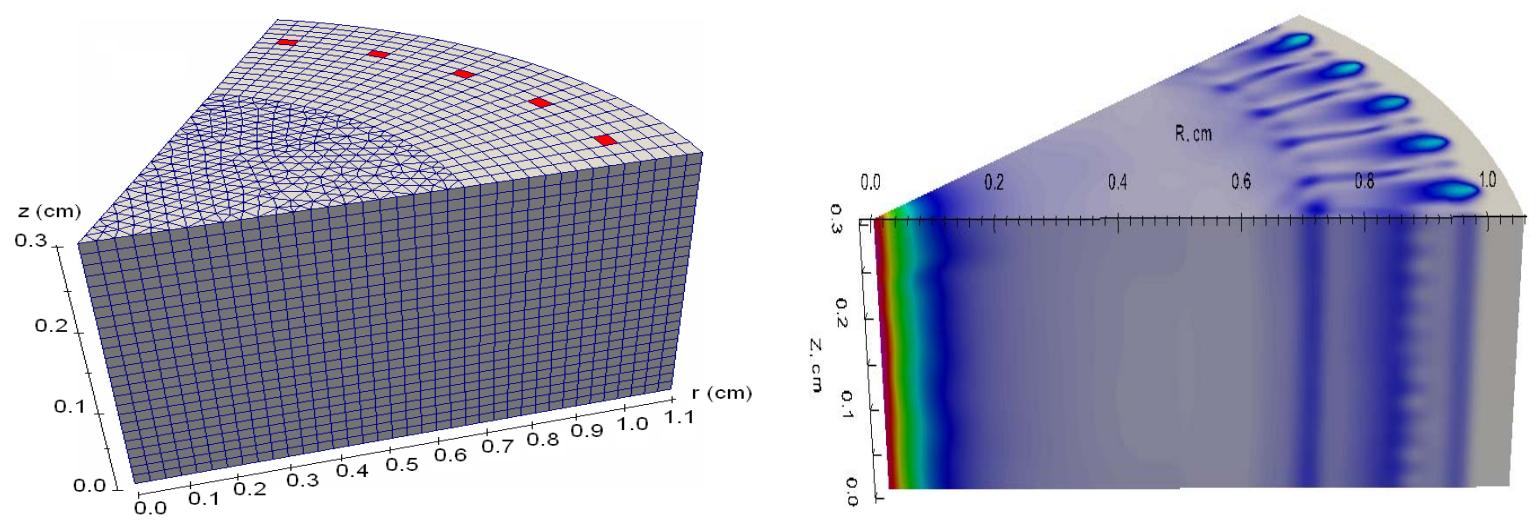

Figure 2. Left: The computational domain (sketch) with the initial positions of the wires. Right: Plasma density distribution in two sections $\left(R-Z\right.$ and $R-\varphi$ planes in cylindrical coordinates, $\left.\mathrm{g} \cdot \mathrm{cm}^{-3}\right)$

The results of a plasma implosion simulation are summarized below.

At the beginning of ablation the main processes are Joule heating and radiation losses. At the temperature $T_{e}^{\sim} 10-20 \mathrm{eV}$, the plasma conductivity increases due to ionization approximately linearly with the temperature, while the emissivity increases faster, approximately by the factor $\sim 2$ [23]. Thus, the condition for the existence of thermal (overheating) instability $[17,24]$ cannot be satisfied. Therefore, at the early stage the temperature perturbations correspond to an initial density perturbation level of $\sim 10 \%$.

By the time $t_{5} \sim 70 \mathrm{~ns}$, the current increases up to $1.5 \mathrm{MA}$, and correspondingly the magnetic pressure increases enough to force the plasma implosion (i.e. active acceleration toward the axis). A shock wave appears at the first phase of acceleration. As some part of energy is spent to ionization and radiation ("ionization-radiation barrier" [17]), the matter compression behind the shock wave front is rather high $\left(p / p_{0} \gtrsim 10\right)$.

Ablation of wires creates inhomogeneous plasma distribution (see Fig. 2, right). The plasma density and magnetic field are modulated in the azimuthal direction. The magnetic force lines bend around the denser areas where the current density increases. The elasticity of the magnetic field lines leads to additional acceleration of plasma.

Noticeable perturbations of density at the outer plasma boundary (Fig. 2, right) produce Rayleigh-Taylor hydromagnetic instability. However, this instability is damped due to sufficiently large aspect ratio of the formed plasma shell, the compressibility of the substance, and smoothing of the energy density gradient of the magnetic field, which is partially transferred together with the plasma to the axis,. The instability of the inner plasma boundary 
is not expressed too.

The inhomogeneity of current density and plasma creates the conditions for the development of overheating (thermal) instability in the regions where the Joule heating surpasses the radiation losses. Estimation of the instability increment according to [17] gives a characteristic overheating time $\tau \sim 10-20 \mathrm{~ns}$. As a result of the instability, at $t \sim 30 \mathrm{~ns}$ the electron temperature in the spots near the plasma boundary is significantly higher than that in the surrounding matter. The computed values are: $T_{e}=45 \mathrm{eV}$ in the spots, and $T_{e}=20 \mathrm{eV}$ in the surrounding plasma.

The thermal instability causes a change in the plasma dynamics. The magnetic field penetrates through the plasma to the skin depth. Due to plasma overheating, the skin layer in the hot spots is much thinner than in the surrounding plasma.

Magnetic force lines take the appearance of "arches" which bend around hot spots. The initial inhomogeneity increases, while the thermal pressure is less than the magnetic one

( $\left.\beta=B^{2} / 8 \pi P \sim 0.1\right)$ due to radiation cooling. Thus the thermal pressure cannot prevent the development of azimuthal perturbations. The distorted shock wave triggers the instability of a strongly radiative thermally inhomodeneous plasma.

By the time $t_{7} \sim 80-90 \mathrm{~ns}$, the plasma velocity in the shock wave reaches $2.2 \cdot 10^{7} \mathrm{~cm} / \mathrm{s}$. Ions are heated up to $T_{i} \sim 1 \mathrm{keV}$, and electron temperature is $T_{e} \sim 200 \mathrm{eV}$. The density/temperature perturbations lead to the magnetic field breakthrough, thus violating the uniformity of a plasma shell compression (Fig. 3). This also causes the development of instability in a "thermally inhomogeneous plasma" $[19,20]$ and the pressure difference reaches $p_{\max } / p_{\min } \sim 2$. The development of non-isothermic instability lasts approximately 20-30ns. The intensive motion leads to equalizing the pressure in the central core and smoothing the other perturbations.

At $t_{7} \sim 90 \mathrm{~ns}$ the first plasma portions reach the axis and the process of stagnation begins. The average parameters of the "near-axis" plasma are the following: velocity $\sim 5 \cdot 10^{7} \mathrm{~cm} / \mathrm{s}$, electron temperature $T_{e} \sim 100 \mathrm{eV}$, ion temperature $T_{i} \sim 300 \mathrm{eV}$, density varies between $\sim 5 \cdot 10^{-2}-10^{-1} \mathrm{~g} / \mathrm{cm}^{3}$, which is in good agreement with the experimental data [25]. Thus, it is shown that the model of prolonged plasma creation [21] correctly describes the rate of plasma input into the region of the forming pinch.

Due to the fast implosion of the evaporated material of the wires and the intense process of radiation cooling of the stagnated plasma, its density in the axial zone significantly exceeds the density in the peripheral zone. The formation of the central pinch replaces the shell structure of the plasma. This process is activated at the time $t_{8} \sim 110 \mathrm{~ns}$, when the current pulse reaches its maximum, and the entire plasma mass moves to the axis. At this point, the stagnation of the plasma bulk is observed, its warming up, and a sharp increase in the soft Xray radiation yield, which is shown in Fig. 4. 

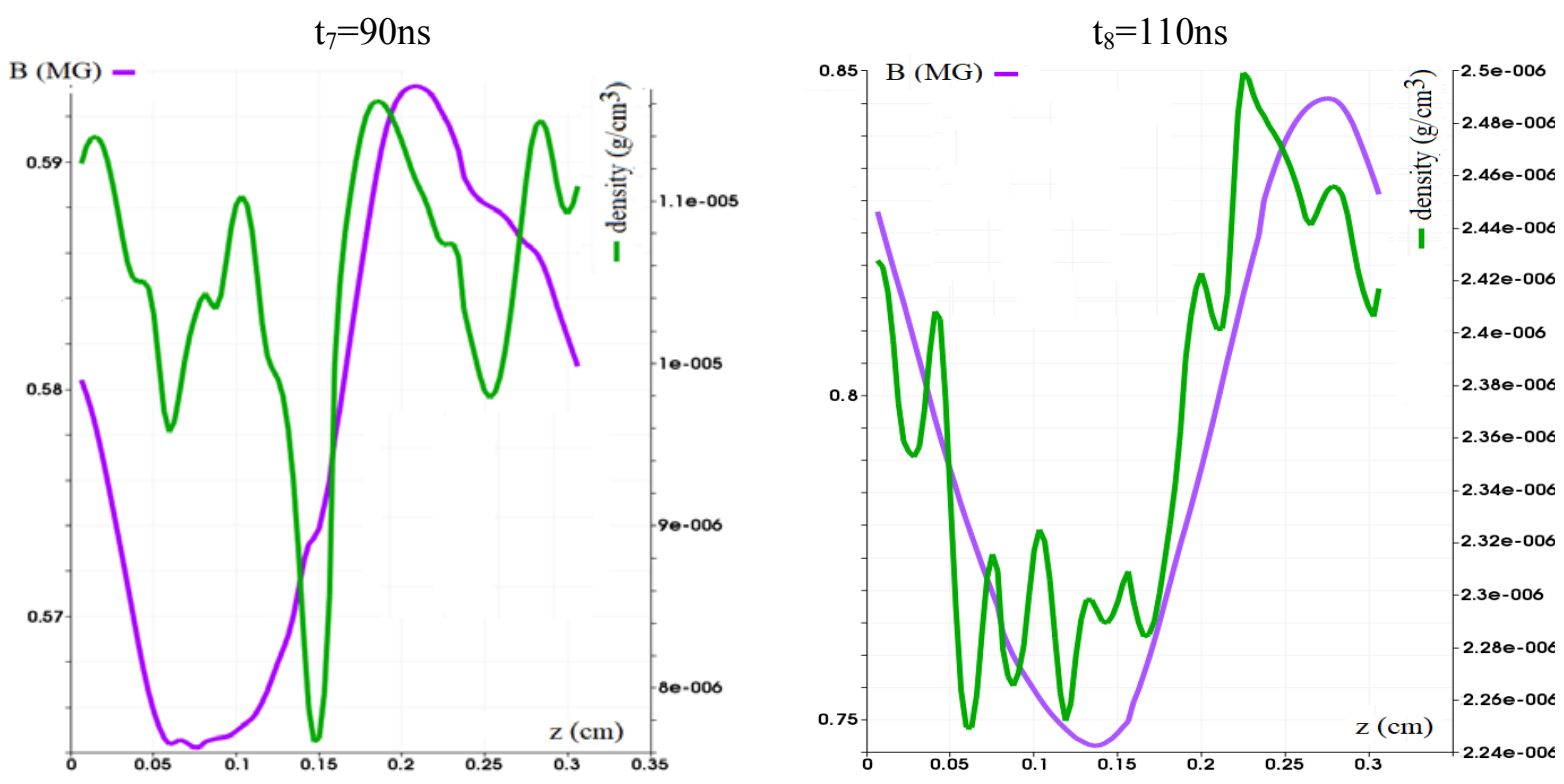

Figure 3. Numerical results: Axial distributions of the azimuthal magnetic field and plasma density inside the wire array at $r_{p}=0.89 \mathrm{~cm}$.
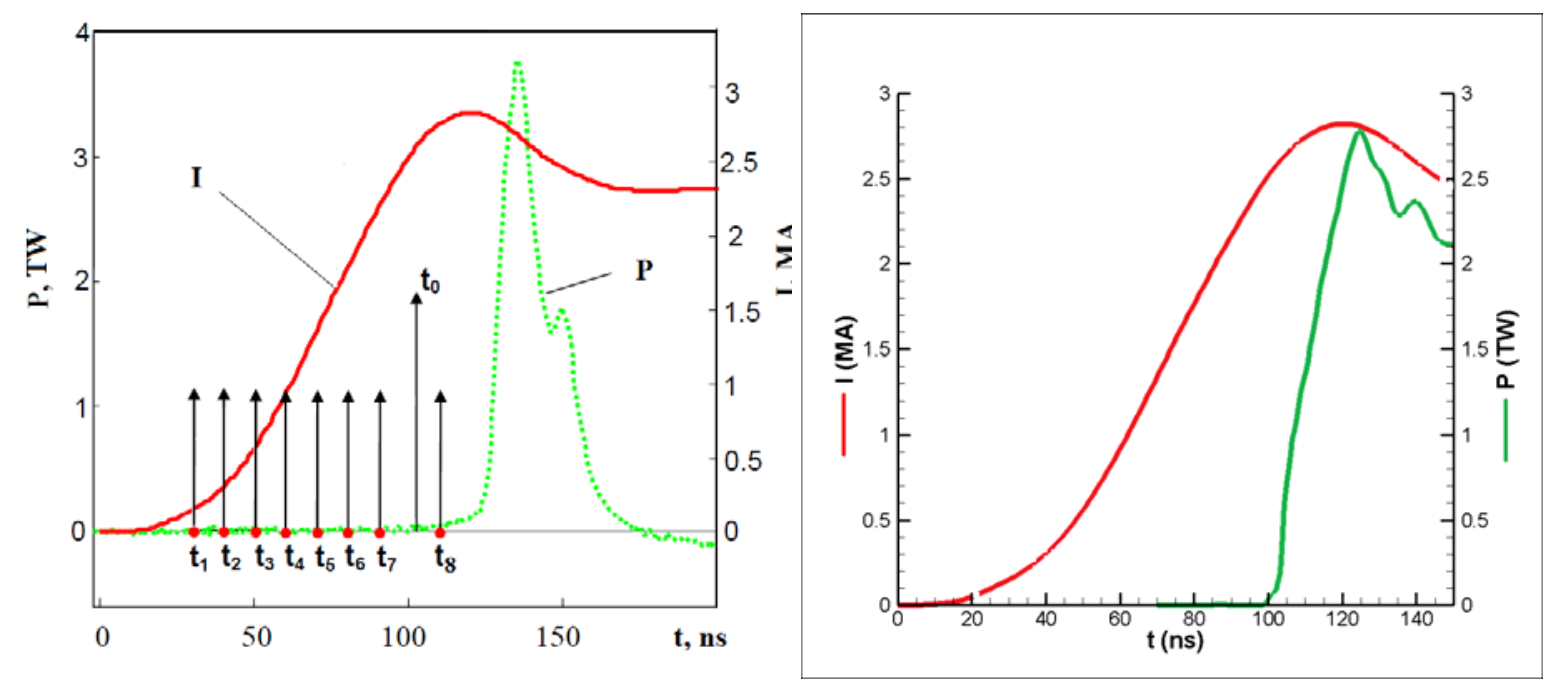

Figure 4. SXR pulse: experiment No 5265 TRINITY [25] (left) and the simulation result (right). $t_{0}$ is the moment of completion of plasma ablation.

\section{CONCLUSIONS}

The methods presented here for solving the equations of the Lagrangian-Eulerian RMHD model were tested by various computational experiments reproducing wave processes in a magnetized medium, e.g. Alfvén waves, magnetosonic waves, decays of MHD discontinuities $[14,27]$, flows with uniform deformation occurring in the vicinity of the zero line of the magnetic field [28], etc. It was shown by computational experiments and application 
simulations, that it is advisable to use the method of physical processes splitting, if the thermal pressure is greater than the magnetic one $p \geq B^{2} / 8 \pi$ during the entire simulated process. In the opposite situation, the method of combined iterations is more resource saving.

The splitting method is easier to implement and allows saving about $40-50 \%$ of arithmetic operations as compared to the combined iterations method. However, when magnetic pressure prevails the thermal one $\left(B^{2} / 8 \pi\right)>p$, the separate accounting of physical processes demands the restriction on the integration time step, similar to that obtained in [14] for the 2D case:

$$
\Delta t<\frac{A h}{\sqrt{\frac{B^{2}}{8 \pi \rho}+(\gamma-2) \frac{p}{\rho}}}, h=V / S_{\max }, A=\text { const } \sim 1 .
$$

Here $V$ is the mesh cell volume, $S_{\max }$ is the maximum value of the side face area.

In simulations of imploding current-carrying plasma accompanied by strongly radiating shock waves, the method of adaptable artificial viscosity [15] appeared to be a resource saving and robust numerical tool. It was indicated, that this method makes possible simulation of transient plasma flows with a significant ion-electron temperature difference. It provides good practical accuracy, which allows comparison with experimental results. Note also that the method is well suitable for the use of real life wide-range EOS.

The developed numerical technique was applied to simulations of Z-pinch implosion at Angara-5-1 facility. The magnetic flux breakthrough into an array made of thin aluminum wires was studied. The numerical results are in good agreement with the experimental data on the basic parameters such as the time when the pinch reached its final state (plasma cumulation near the axis of the system) and the soft x-ray radiation power. Thus we can conclude that it is possible to use the proposed method based on the completely conservative difference scheme for solving plasma dynamics problems, and carry out predictive simulation of experiments with plasma accelerated by electromagnetic force produced by a powerful current pulses. At the same time, the numerical simulation substantially supplements the experimental data, since it provides information on the dynamics of magnetic implosion, which cannot be obtained in the experiment due to the limited capabilities of diagnostics.

Let us take up the liner implosion in cylindrical coordinates. Then, in the $(R-z)$ plane perpendicular to the azimuthal magnetic field force lines, the Rayleigh-Taylor instability causes the most serious perturbations. In the $(R-\varphi)$ plane, the instabilities of thermally inhomogeneous plasma are of importance due to disbalances of Joule heating and radiation losses. For further clarification of the instability effect, we need a detailed examination of the initial perturbations evolution by individual harmonics in a certain spectral range, including perturbations of an arbitrary form (superposition of harmonics), various initial amplitudes, transition to a nonlinear stage, etc. Here we concentrate on the fact that although the problem of instability in many cases is considered as "purely mechanical", the energy aspect is very important concerning the dynamics of Z-pinch plasmas. The rate of instability depends on the rate of plasma acceleration as well as on the aspect ratio of a plasma shell formed due to wire ablation. The process in whole is determined by the energy exchange between the electromagnetic field and the plasma, as well as the energy balance in the plasma, where the radiation transfer is the largest contributor. The performed simulation, even with a mild Rayleigh-Taylor instability, shows that at the final stage of compression, the distribution of density and temperature is substantially homogeneous in space. The radiation absorption 
coefficient, which is inversely proportional to the mean free path, varies by several orders of magnitude in the computational domain. The radiation is locked in the central region of the pinch, as a result it radiates like a surface source.

The computations were carried out on the supercomputers MVS-100K (JSCC RAS) and K100 (KIAM RAS).

\section{APPENDIX}

The equation of total energy balance for the coupled plasma - electromagnetic field system:

$$
\begin{aligned}
& \widehat{E}_{t o t}-E_{t o t}=-\Delta t \sum_{m l n}\left(\widehat{G}_{e_{m l n}}+\widehat{G}_{i_{m l n}}\right) \widehat{V}_{m l n}+
\end{aligned}
$$

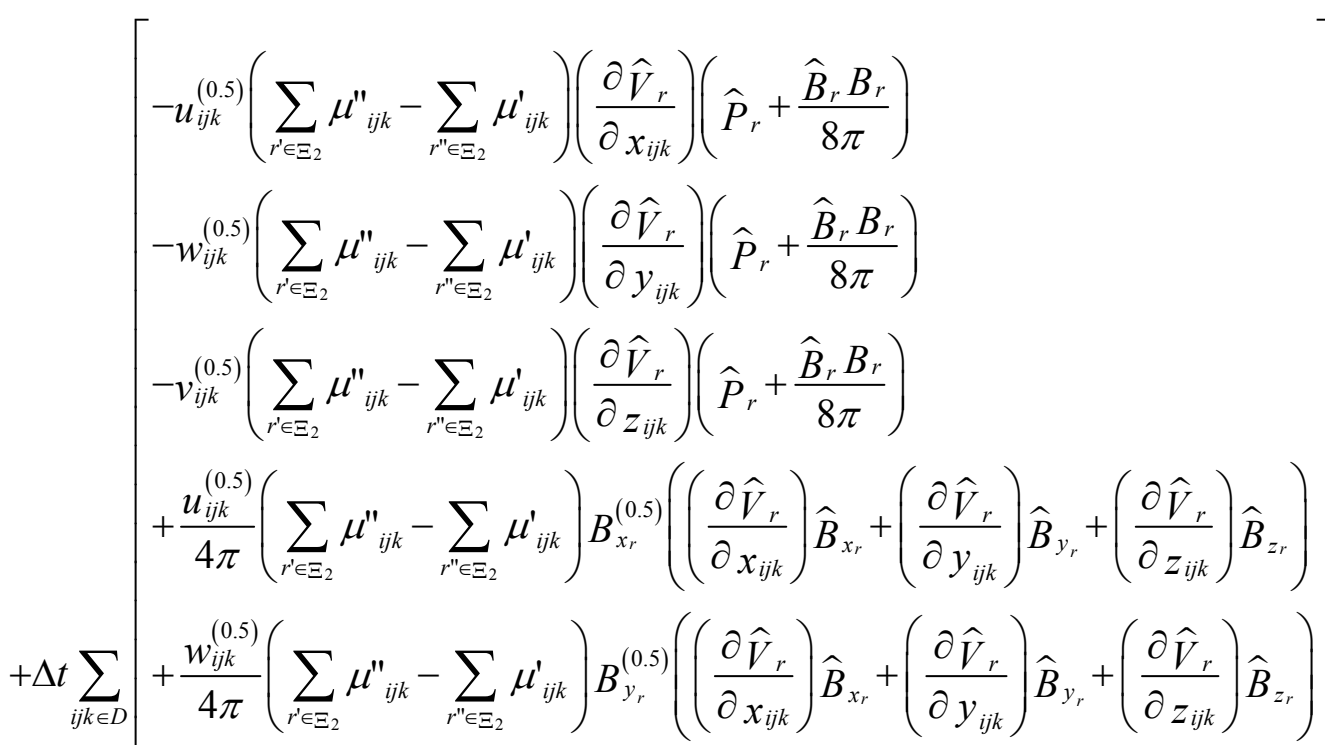

$$
\begin{aligned}
& +\frac{v_{i j k}^{(0.5)}}{4 \pi}\left(\sum_{r^{\prime} \in \Xi_{2}} \mu^{\prime \prime}{ }_{i j k}-\sum_{r^{\prime \prime} \in \Xi_{2}} \mu_{i j k}^{\prime}\right) B_{z_{r}}^{(0.5)}\left(\left(\frac{\partial \hat{V}_{r}}{\partial x_{i j k}}\right) \widehat{B}_{x_{r}}+\left(\frac{\partial \widehat{V}_{r}}{\partial y_{i j k}}\right) \widehat{B}_{y_{r}}+\left(\frac{\partial \widehat{V}_{r}}{\partial z_{i j k}}\right) \widehat{B}_{z_{r}}\right) \\
& +\frac{\widehat{E}_{x_{i j k}}}{4 \pi}\left(\sum_{r^{\prime} \in \Xi_{2}} \eta_{i j k}^{\prime \prime}-\sum_{r^{\prime} \in \Xi_{2}} \eta_{i j k}^{\prime}\right)\left(\left(\frac{\partial \widehat{V}_{r}}{\partial y_{i j k}}\right) \widehat{B}_{z_{r}}^{(0.5)}-\left(\frac{\partial \widehat{V}_{r}}{\partial z_{i j k}}\right) \widehat{B}_{y_{r}}^{(0.5)}\right) \\
& +\frac{\widehat{E}_{y_{i j k}}}{4 \pi}\left(\sum_{r^{\prime} \in \Xi_{2}} \eta^{\prime \prime}{ }_{i j k}-\sum_{r^{\prime \prime} \in \Xi_{2}} \eta_{i j k}^{\prime}\right)\left(\left(\frac{\partial \widehat{V}_{r}}{\partial z_{i j k}}\right) \widehat{B}_{x_{r}}^{(0.5)}-\left(\frac{\partial \widehat{V}_{r}}{\partial x_{i j k}}\right) \widehat{B}_{z_{r}}^{(0.5)}\right) \\
& +\frac{\widehat{E}_{z_{i j k}}}{4 \pi}\left(\sum_{r^{\prime} \in \Xi_{2}} \eta_{i j k}^{\prime \prime}-\sum_{r^{\prime \prime} \in \Xi_{2}} \eta_{i j k}^{\prime}\right)\left(\left(\frac{\partial \widehat{V}_{r}}{\partial x_{i j k}}\right) \widehat{B}_{y_{r}}^{(0.5)}-\left(\frac{\partial \widehat{V}_{r}}{\partial y_{i j k}}\right) \widehat{B}_{x_{r}}^{(0.5)}\right)
\end{aligned}
$$




\section{REFERENCES}

[1] A.A. Samarskii, The theory of difference schemes, New York - Basel. Marcel Dekker, Inc, (2001).

[2] G.I. Marchuk, Metody rasshscepleniia, Moscow: Nauka (1988).

[3] A. K. Alekseev, A.E. Bondarev, A.E. Kuvshinnikov, "Comparative analysis of the accuracy of OpenFoam solvers for the oblique shock wave problem", Math. Montis, 45, 95-106 (2019).

[4] I.N. Konshin, K.M. Terekhov and Yu.V. Vassilevski, "Numerical modeling via INMOST software platform", Math. Montis, 47, 75-86 (2020).

[5] A.A. Samarskii, Yu.P. Popov, Raznostnye metody resheniia zadach gazovoi dinamiki, Moscow: Nauka (1992).

[6] V.P. Smirnov, "Fast liners for inertial fusion", Plasma Phys. Controlled Fusion, 33, 1697 (1991).

[7] Michael A. Liberman, John S. De Groot, Arthur Toor, Rick B. Spielman, Physics of highdensity Z-pinch plasmas, Springer (1998).

[8] James J. Duderstadt, Gregory A. Moses, Inertial Confinement Fusion, John Wiley and Sons, New York (1982).

[9] V.I. Oreshkin, Radiation of High-temperature Plasma. Pinch-effect, LAP Lambert Academic Publishing, Saarbrukken (2012).

[10] Y. Zhang, U. Shumlak, B. A. Nelson, R. P. Golingo, T. R. Weber, A. D. Stepanov, E. L. Claveau, E. G. Forbes, Z. T. Draper, J. M. Mitrani, H. S. McLean, K. K. Tummel, D. P. Higginson and C.M. Cooper, "Sustained Neutron Production from a Sheared-Flow Stabilized Z - Pinch", Phys. Rev. Lett., 122, 135001 (2019). doi:10.1103/PhysRevLett.122.135001.

[11] M. R. Gomez, et al, "Assessing Stagnation Conditions and Identifying Trends in Magnetized Liner Inertial Fusion". IEEE Transactions on Plasma Science. IEEE Transactions on Plasma Science, 47(5), 2081-2101 (2019).

[12] V.V. Aleksandrov, A.V. Branitski, E.V. Grabovskiy, A.N. Gritsuk, K.N. Mitrofanov, I.N. Frolov, V.A. Gasilov, O.G. Olkhovskaya, P.V. Sasorov, "Study of interaction between plasma flows and the magnetic field at the implosion of nested wire arrays", Plasma Physics and Controlled Fusion, 61 (3), 035009 (2019).

[13] G.A Kulikovskiy, A.G Lyubimov, Magnitnaya gidrodinamika, Moscow: Logos (2011).

[14] A.Yu. Krukovskiy, V.A. Gasilov, Yu.A. Poveschenko, Yu. S. Sharova, L.V. Klochkova, "Implementation of the iterative algorithm for numerical solution of 2D magnetogasdynamics problems", Matem. Mod., 32 (1), 50-70 (2020). doi: 10.20948/mm-2020-01-04.

[15] I.V. Popov, I.V. Friazinov, Metod adaptivnoi iskusstvennoi viazkosti chislennogo resheniia uravnenii gazovoi dinamiki, Moscow: Krasand (2015).

[16] C.W. Hirt, A.A. Amsden and J.L. Cook, "An arbitrary Lagrangian-Eulerian computing method for all ow speeds", J. Comput. Phys., 14, $227-253$ (1974).

[17] A.A. Samarskii, A.P. Mikhailov, Principles of Mathematical Modeling. Ideas, Methods, Examples, London and New York. Taylor and Francis, (2002).

[18] A.A. Samarskii, A.V. Gulin, Chislennye metody, Moscow: Nauka (1989).

[19] R. Benattar, P. Ney, A. Nikitin, S.V. Zakharov, A.A. Otochin, A.N. Starostin, A.E. Stepanov, A.F. Nikiforov, V.G. Novikov, A.D. Solomyannaya, V.A. Gasilov and A.Yu. Krukovskii, "Implosion Dynamics of a Radiative Z-Pinch", IEEE Transactions on Plasma Science, 26 (4) (special issue on Z-pinch plasmas), 1210-1223 (1998).

[20] A.S. Boldarev, E.A. Bolkhovitinov, I.Yu. Vichev, G.S. Volkov, V.A. Gasilov, E.V. Grabovskii, A.N. Gritsuk, S.A. Dan'ko, V.I. Zaitsev, V.G. Novikov，G.M. Oleinik，O.G. Olkhovskaya, A.A. Rupasov, M.V. Fedulov, A.S. Shikanov, "Methods and Results of Studies of the 
Radiation Spectra of Megampere Z-Pinches at the Angara-5-1 Facility", Plasma Physics Reports, 41 (2), 178-181 (2015).

[21] V. V. Aleksandrov, A.V. Branitskii, G.S. Volkov, E.V. Grabovskii, M.V. Zurin, S.L. Nedoseev, G.M. Oleinik, A.A. Samokhin, P.V. Sasorov, V.P. Smirnov, M.V. Fedulov, I.N. Frolov, "Dynamics of Heterogeneous Liners with Prolonged Plasma Creation", Plasma Physics Reports, 27 (2), 89-109 (2001).

[22] V.A. Gasilov, A.S. Boldarev, S.V. D'yachenko, O.G. Olkhovskaya, E.L. Kartasheva, S.N. Boldyrev, G.A. Bagdasarov, I.V. Gasilova, M.S. Boyarov, V.A. Shmyrov, "Program package MARPLE3D for simulation of pulsed magnetically driven plasma using high performance computing", Matem. Mod., 24 (1), 55-87 (2012).

[23] A.F. Nikiforov, V.G. Novikov, V.B. Uvarov, Quantum-Statistical Models of Hot Dense Matter. Methods for Computation Opacity and Equation of State, Basel, Berlin: Birkhauser Verlag (2005).

[24] V.V. Alexandrov, E.V. Grabovsky, M.V. Zurin, I.V. Krasovsky, K.N. Mitrofanov, S.L. Nedoseev, G.M. Oleinik, I.Yu. Porofeev, A.A. Samokhin, V.P. Smirnov, M.V. Fedulov, I.N. Frolov, P.V. Sasorov, "Characteristics of high-power radiating imploding discharge with cold start" Journal of Experimental and Theoretical Physics, 99 (6), 1150-1172 (2004).

[25] K.N. Mitrofanov, V.V. Aleksandrov, E.V. Grabovski, E.A. Ptichkina, A.N. Gritsuk, I.N. Frolov, Y.N. Laukhin, "Study of the termination phase of plasma production and the formation of magnetic flux breakthroughs during wire array implosion", Plasma Physics Reports, 40 (9), 779-806 (2014).

[26] A. A. Samarskii, "Some results of the theory of difference methods.", Differentsial'nye Uravneniya, 16 (7), 1155-1171, 1348 (1980).

[27] K.V. Brushlinskiy, Matematicheskiye $i$ vychislitel'nyye zadachi magnitnoy gazodinamiki, Moscow: BINOM (2020). (in Russian)

[28] E. Priest and T. Forbes, Magnetic Reconnection, Cambridge, UK: Cambridge University Press, (2007).

[29] Linear Solver: https://trilinos.github.io/linear_solver.html (Accessed December 19, 2020)

[30] SALOME: http://www.salome-platform.org/ (Accessed December 19, 2020)

[31] ParaView: https://www.paraview.org/ (Accessed December 19, 2020)

[32] THERMOS: http://keldysh.ru/cgi/thermos/navigation.pl?en,home (Accessed December 19, 2020)

Received December 28, 2020 